\title{
Redes de Sensores sem Fio Aplicadas à Fisioterapia: Implementação e Validação de um Sistema de Monitoramento de Amplitude de Movimento
}

\author{
Renan C. A. Alves ${ }^{1}$, Cíntia B. Margi ${ }^{1}$, \\ Fabíola C. L. dos Santos ${ }^{2}$, Bruno T. de Oliveira ${ }^{1}$ \\ ${ }^{1}$ Laboratório de Arquitetura e Redes de Computadores \\ Departamento de Engenharia de Computação e Sistemas Digitais \\ Escola Politécnica - Universidade de São Paulo \\ São Paulo - SP - Brasil \\ ${ }^{2}$ Departamento de Fisioterapia, Fonoaudiologia e Terapia Ocupacional \\ Faculdade de Medicina - Universidade de São Paulo \\ São Paulo - SP - Brasil \\ \{renanalves, cintia, fasantos, brunotrevizan\}@usp.br
}

\begin{abstract}
Range of motion is an important parameter used on evaluation and physical therapy treatment. Systems used currently have limited operation capabilities. This work's main contribution is the specification, implementation and validation of a real time wireless sensor network based system for joint angle monitoring: FlexMeter. Sensing nodes gather joint angle information through accelerometers, while wireless devices provide mobility to patient and physical therapist. System validation has three steps: sensor measurement validation, usability validation and knee joint measurement validation. Validation results show that system use could subserve physical therapist's work.
\end{abstract}

Resumo. A amplitude de movimento é um parâmetro importante utilizado na avaliação e durante o tratamento fisioterapêutico. Sistemas disponíveis e utilizados atualmente possuem atuação limitada. A principal contribuição deste trabalho é a especificação, implementação e validação de um sistema de monitoramento, em tempo real, de ângulos articulares baseado em redes de sensores sem fio: FlexMeter. Os nós sensores utilizam acelerômetros para obter as informações de ângulos articulares, e a rede sem fio dá mobilidade ao paciente e ao fisioterapeuta. A validação do sistema incluiu três etapas: validação das medidas com os sensores, validação de usabilidade, e validação de medidas em articulação de joelho. Os resultados da validação demonstraram como o uso do sistema pode facilitar o trabalho do fisioterapeuta.

\section{Introdução}

As grandes evoluções da medicina costumam acompanhar as evoluções tecnológicas, caracterizando um processo multidisciplinar, envolvendo principalmente as ciências médicas e a engenharia. Um dos principais fatores deste processo de evolução são os instrumentos de mensuração, que permitem um monitoramento do estado de saúde do paciente, facilitando as decisões dos profissionais da área saúde. O advento da tecnologia da informação e a utilização dos sistemas de informação, possibilitam uma análise mais refinada 
dos dados dos pacientes e auxiliam na manutenção do histórico médico de cada indivíduo. No domínio de conhecimento da saúde, a fisioterapia mostra-se uma área carente destes aplicativos e ferramentas.

A fisioterapia é uma ciência da saúde que estuda, previne e trata os distúrbios cinéticos funcionais intercorrentes em órgãos e sistemas do corpo humano, gerados por alterações genéticas, por traumas e por doenças adquiridas. Esta fundamenta suas ações em mecanismos terapêuticos próprios [COFFITO 2012].

A goniometria, ou mensuração dos ângulos de articulações, é uma tarefa comum a profissionais da área de fisioterapia, tratando-se de uma métrica importante para quantificar a evolução do quadro de seus pacientes [Marques 2003]. As ferramentas existentes, como o goniômetro e outras apresentadas na Seção 2.2, requerem que o profissional interrompa a sessão para a obtenção de medições necessárias, e demandam considerável trabalho manual para o registro dos dados obtidos. A adoção de sistemas de informação em conjunto com mecanismos automatizados de coleta dos dados permite ao profissional concentrar seus esforços e atenção no paciente, focando na análise dos dados de evolução e na orientação dos exercícios.

As redes de sensores sem fio (RSSFs) são comumente utilizadas em aplicações de monitoramento e podem ser definidas como uma classe especial de redes ad hoc de múltiplos saltos $\left(\right.$ MANETs $\left.^{1}\right)$, caracterizadas por ser sem fio e pela ausência de infraestrutura fixa [Margi 2006]. Tipicamente, os nós participantes possuem baixa capacidade de processamento, pouca memória para armazenamento, sistemas de comunicação de baixa velocidade, largura de banda limitada e fonte de energia escassa [Culler et al. 2004].

A principal contribuição deste trabalho é a especificação e desenvolvimento de um sistema de monitoramento de ângulos articulares baseado em RSSF. Este sistema permite ao fisioterapeuta a mensuração e a visualização de ângulos articulares em tempo real, sem a necessidade de interromper a sessão de terapia para realizar mensurações. Os dados coletados são armazenadas, permitindo a visualização da sessão e sua análise posterior.

Através do estudo da evolução das medidas é possível construir um indicador da efetividade dos exercícios e de possíveis dificuldades encontradas pelos pacientes, permitindo o acompanhamento e a supervisão dos respectivos tratamentos. Assim, este projeto almeja contribuir para área de fisioterapia no que diz respeito a sistemas de monitoramento, descrevendo o projeto, implementação e validação de um protótipo do sistema FlexMeter.

O presente trabalho dá continuidade ao artigo apresentado no SBSI 2012 [Alves et al. 2012], incluindo uma versão estendida da descrição do sistema e uma nova seção de resultados de validação em pacientes.

Este trabalho está organizado da seguinte forma. A Seção 2 trata dos trabalhos relacionados. A Seção 3 especifica e detalha as características de hardware e software do sistema FlexMeter, aqui apresentado. Em seguida, os resultados da validação de medidas, tanto com ângulos de referência como em articulações, e usabilidade do sistema são apresentados e discutidos na Seção 4, enquanto a Seção 5 apresenta as considerações finais e trabalhos futuros.

\footnotetext{
${ }^{1}$ Multihop Ad hoc NETworks
} 


\section{Trabalhos Relacionados}

Os trabalhos relacionados discutidos nesta seção podem ser divididos em duas categorias, de acordo com sua proximidade a engenharia (Seção 2.1) ou fisioterapia (Seção 2.2).

\subsection{Redes de Sensores Sem Fio}

Uma família de aplicações de RSSF que se destaca é a de eHealth, um termo geral utilizado para descrever o uso combinado de eletrônica, comunicação e sistemas de informação provendo suporte ao setor da saúde [Della Mea 2001]. O uso de RSSF permite aplicações de monitoramento mais robustas, devido a diversos contextos pessoais que podem ser capturados e usados para prover melhores serviços de saúde, como emergência, cuidado com idosos, e auxílio a fisioterapia.

O projeto CodeBlue [Malan et al. 2004] visa oferecer infraestrutura de comunicação sem fio para variados cenários de saúde, com aplicação em ambiente hospitalar, triagem de pacientes, situações de desastre, entre outros. O projeto Mercury [Lorincz et al. 2009] é uma plataforma wearable baseada em RSSF para análise de movimento de pacientes em tratamento de doenças neuromotoras, como Mal de Parkinson, epilepsia e derrame cerebral.

Silva et al. (2007) propôs o monitoramento dos batimentos cardíacos do paciente e a sua postura em sessões de hidroterapia. A postura é monitorada através de ângulos rotacionais dos membros do corpo com sensores interligados com cabos. Os dados são enviados a estação base por um módulo sensor flutuante, de modo a evitar a comunicação sem fio subaquática. Tal trabalho tem como objetivo medir apenas a posição da coluna, e não do ângulo de movimento de outros membros.

O'donovan et al. (2007) descreve a teoria para se realizar mensuração de ângulos com redes de sensores através do uso de acelerômetros, giroscópios e magnetômetros. $\mathrm{O}$ mesmo autor propôs uma prova de conceito para medidas de ângulos de joelho e tornozelo, não mencionando a existência de um sistema de informação para auxiliar a análise dos dados obtidos [O’Donovan and Ayer 2011].

\subsection{Instrumentação de Fisioterapia}

A amplitude de movimento $(\mathrm{ADM})^{2}$ é um importante parâmetro utilizado na avaliação e durante o tratamento fisioterapêutico. Muitas vezes a avaliação da amplitude do movimento faz parte da definição do prognóstico de um indivíduo submetido a fisioterapia [Portney and Watkins 2000].

A ADM varia de indivíduo para indivíduo de acordo com a idade, sexo, prática de atividade física, presença ou ausência de disfunção e o grau de força muscular quando o indivíduo é submetido à avaliação da ADM ativa [Rothstein 1985]. A padronização da metodologia empregada é de extrema importância uma vez que pode evitar possíveis vieses.

Alguns instrumentos vêm sendo utilizados para tal mensuração. O mais utilizado é o goniômetro universal (Figuras 1(a) e 2(a)), por ser de fácil utilização e baixo custo. Este instrumento é composto por duas hastes, denominadas braços, articuladas em um

\footnotetext{
${ }^{2}$ Quantidade de movimento passiva ou ativa que paciente consegue realizar, medida em graus [Amado and Maria 2006]
} 


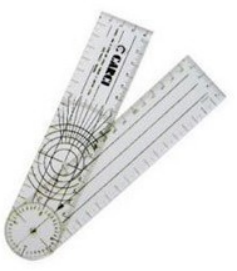

(a) Goniômetro

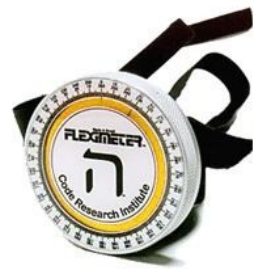

(b) Flexímetro

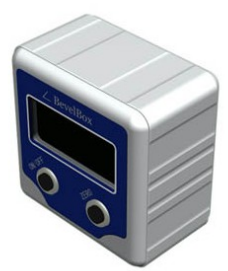

(c) Inclinômetro

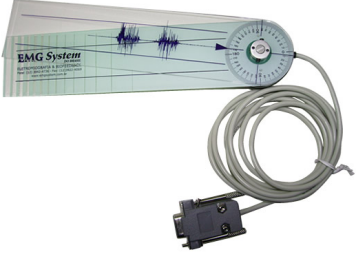

(d) Eletrogoniômetro

Figura 1. Instrumentos ${ }^{3}$

disco graduado que indica o valor do ângulo medido. Para a mensuração é necessário a utilização de pontos anatômicos determinados [Gajdosik and Bohannon 1987].

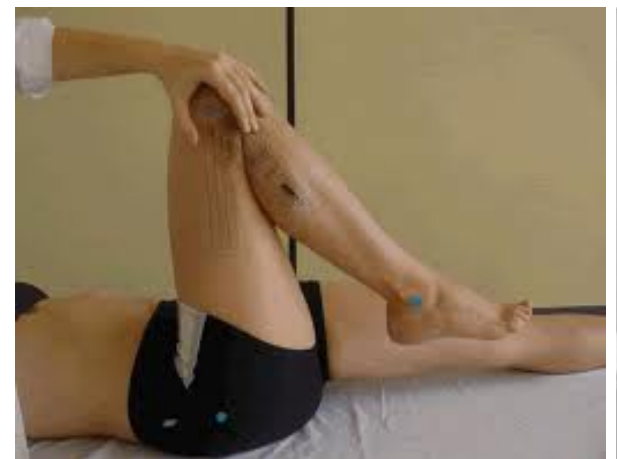

(a) Goniômetro

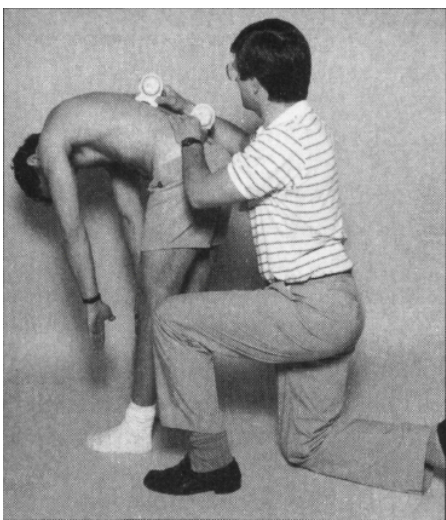

(c) Inclinômetro

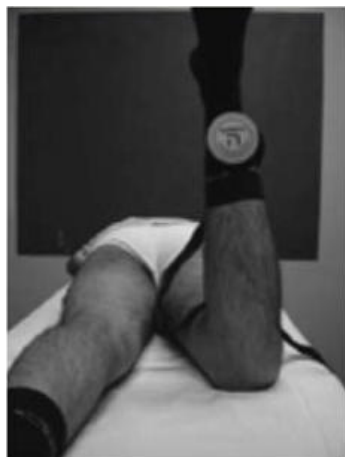

(b) Flexímetro

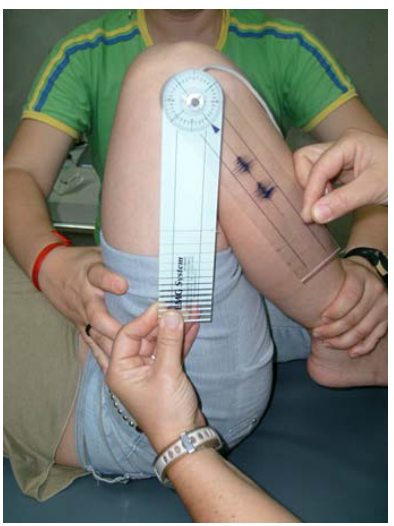

(d) Eletrogoniômetro

Figura 2. Instrumentos em uso ${ }^{4}$

Outro instrumento é o flexímetro (Figuras 1(b) e 2(b)), cujo sistema pendular gravitacional possui indicação do ângulo produzida por efeito da gravidade, minimizando os erros de interpretação do eixo longitudinal correspondente. A escala angular é desenhada com incrementos de $1^{\circ}$ a $360^{\circ}$, progressivos e regressivos. As vantagens da utilização

\footnotetext{
${ }^{3}$ Figuras 1(a) e Figura 1(c) retiradas de [Fysio Brasil 2012]. Figura 1(b) retirada de [CardioMed 2012]. Figuras 1(d) retirada de [EMG System do Brail 2012].

${ }^{4}$ Figura 2(a) retirada de [Marques 2003]. Figura 2(b) retirada de [Canto et al. 2005]. Figura 2(c) retirada de [Williams et al. 1993]. Figura 2(d) retirada de [Batistela 2008]
} 
do flexímetro, segundo [Clarkson and Gilewich 1991], em relação a um goniômetro universal incluem o fato de não ser necessário alinhar o flexímetro com o eixo articular; a facilidade de medir os movimentos rotacionais e a ocorrência de pouca mudança no alinhamento do aparelho segundo os fabricantes.

O inclinômetro digital (Figuras 1(c) e 2(c)) é um instrumento de funcionamento dependente da gravidade através de um sensor que capta medida da angulação. É um instrumento pouco difundido na fisioterapia com um custo mais elevado e independe de pontos anatômicos como o goniômetro universal, embora apresente grande variação de resultados entre avaliadores [Bennell et al. 1998, de Winter et al. 2004].

O eletrogoniômetro (Figuras 1(d) e 2(d)) é um instrumento que permite mensurar a ADM instantânea (assim com o goniômetro universal) ou, em alguns modelos, é possível realizar medições em tempo real. Entretanto, múltiplos instrumentos podem ser necessários para medir diferentes ângulos articulares [Biometrics Ltd 2012] e o posicionamento do instrumento pode interferir significativamente no resultado [Carnaz 2008].

O BioFeed [BioSmart 2012] é um equipamento que propõe monitorar movimentos do corpo humano, através da mensuração da inclinação dos membros do corpo em relação ao solo. Contudo, informações técnicas ou de validação em pacientes não foram encontradas no site [BioSmart 2012].

\section{FlexMeter}

O sistema FlexMeter, cuja proposta inicial foi realizada como projeto de formatura [Polizel et al. 2011], tem como objetivo o acompanhamento em tempo real para a supervisão do tratamento de pacientes de fisioterapia.

Seis dispositivos, chamados módulos sensores, munidos de sensores e transmissores sem-fio são acoplados ao paciente. Através desta RSSF, medem-se ângulos articulares de um paciente ao longo de uma sessão de fisioterapia. Estes dados são enviados e exibidos na estação da fisioterapeuta quantitativamente, através do valor numérico e de gráficos em função do tempo, e qualitativamente, através de um modelo de computação gráfica do paciente, como observado na Figura 3.

Dados característicos de cada sessão, tais como o valor máximo, mínimo e médio

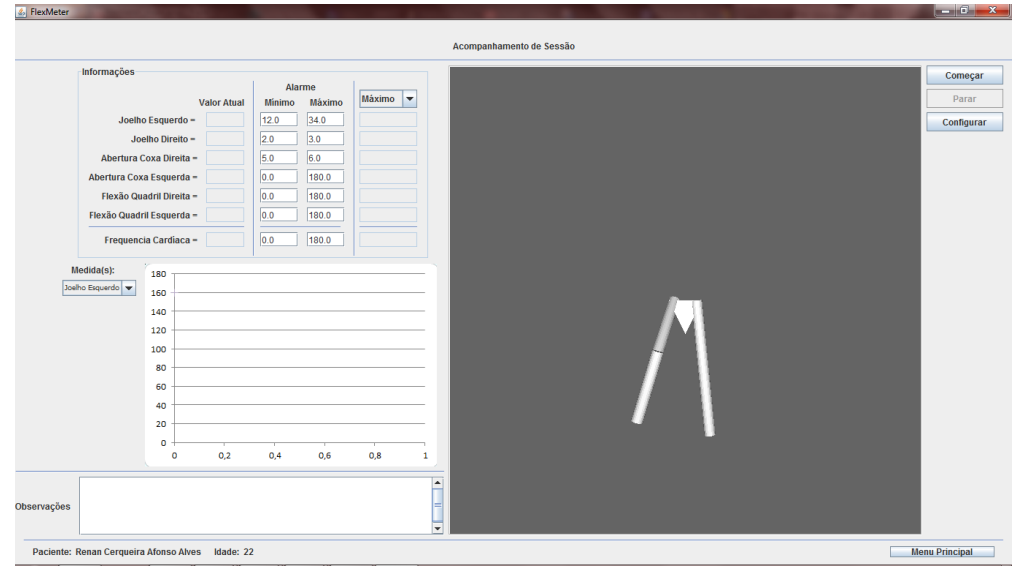

Figura 3. FlexMeter - Tela de acompanhamento 
de cada ângulo medido, são armazenados após o término da sessão. Estes podem ser consultados e analisados, tanto individualmente a cada sessão como através de um gráfico composto por valores de várias sessões, conforme ilustrado na Figura 4.

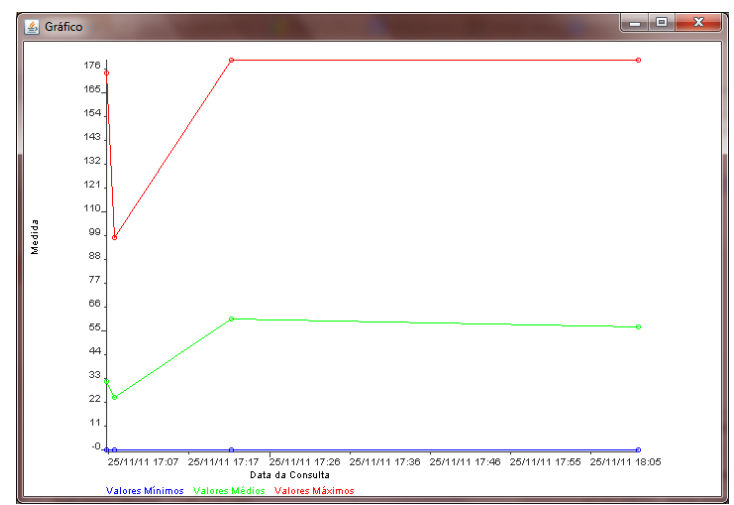

Figura 4. FlexMeter - Dados históricos

No protótipo construído foram contemplados somente ângulos articulares relativos a membros inferiores. A Figura 5 exibe a arquitetura do sistema, indicando o posicionamento dos seis módulos sensores no paciente e a conexão do módulo sorvedouro com a estação do fisioterapeuta, onde o software de processamento de dados é executado.

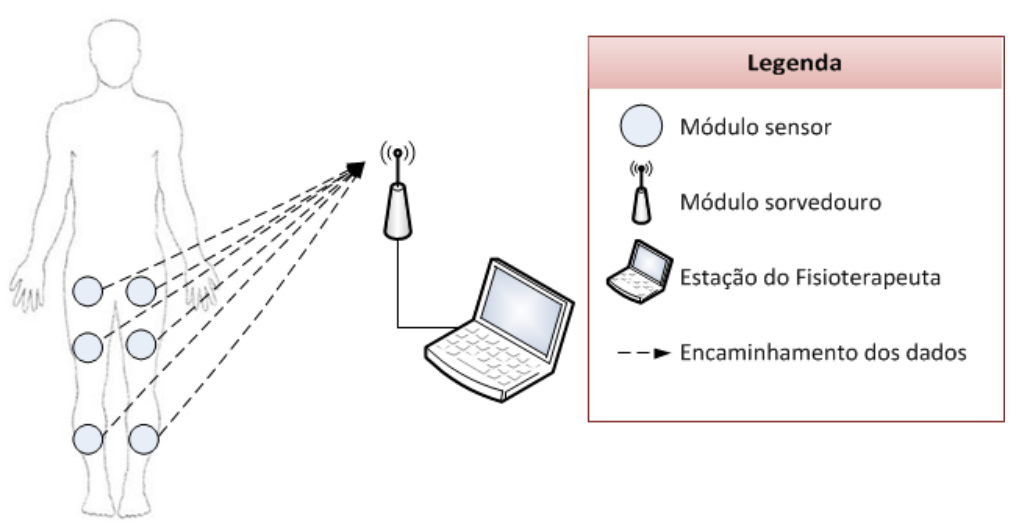

Figura 5. Arquitetura do Sistema

As subseções seguintes descrevem o procedimento utilizado para a realização dos cálculos de ângulos e os componentes de hardware e software que compõem o sistema.

\subsection{Procedimento de cálculos de ângulos}

O ângulo $\theta$ formado por dois vetores, $v_{1}$ e $v_{2}$, em espaço euclidiano pode ser calculado a partir de suas coordenadas em uma base ortonormal da seguinte forma:

$$
\theta=\arccos \left(\frac{v_{1} \cdot v_{2}}{\left|v_{1}\right|\left|v_{2}\right|}\right)
$$

Assim, para determinar ângulos articulares de um paciente, basta determinar vetores que correspondam as linhas de mensuração dos ângulos em uma base comum. Estes vetores podem ser determinados através de módulos sensores acoplados ao paciente. 
Para que seja possível o cálculo da direção de um sensor no espaço, algumas premissas são consideradas:

- a base $B=\left(b_{x}, b_{y}, b_{z}\right)$ do ambiente, comum a todos os módulos sensores, é fixa. Convenciona-se que $b_{z}$ é um vetor unitário no sentido da gravidade, $b_{x}$ é um vetor horizontal unitário perpendicular ao primeiro e $b_{y}$ é obtido através do produto vetorial dos primeiros;

- os módulos sensores possuem uma base ortogonal intrínseca variável em relação a base do ambiente, e suas medidas são feitas em relação a esta base;

- os módulos sensores possuem dispositivos para aferir as componentes da base do ambiente $\left(b_{z}\right.$ e $\left.b_{x}\right)$ em sua base intrínseca;

- um dos eixos da base dos sensores deve estar alinhado com o ponto anatômico a ser observado. Convenciona-se que seja o eixo X.

Desta forma, o problema do cálculo da direção de um sensor no espaço se resume a calcular a direção do eixo $\mathrm{X}$ da base dos sensores na base do ambiente.

Sejam $u=\left(u_{x}, u_{y}, u_{z}\right)$ e $v=\left(v_{x}, v_{y}, v_{z}\right)$ os vetores normalizados que representam os valores medidos por um módulo sensor de $b_{x}$ e $b_{z}$. Portanto, $v$ representa a direção da gravidade, enquanto que $u$ representa o vetor de referência horizontal, ambos representados na base do módulo sensor.

A matriz de mudança de base $M_{s a}$ é a matriz que, se multiplicada pela direita por um vetor, transforma suas coordenadas da base do ambiente para coordenadas na base do módulo sensor. Desta forma, a Equação 2 relaciona a referência horizontal da base do ambiente ao vetor $u$. Analogamente, a Equação 3 relaciona o vetor unitário na direção da gravidade ao valor aferido pelo módulo sensor. Por fim, a Equação 4 os respectivos produtos vetoriais $^{5}$ (a Equação 5 exibe o produto vetorial por extenso).

$$
\begin{aligned}
M_{s a} \cdot(1,0,0) & =\left(u_{x}, u_{y}, u_{z}\right) \\
M_{s a} \cdot(0,0,1) & =\left(v_{x}, v_{y}, v_{z}\right) \\
M_{s a} \cdot(0,1,0) & =u \times v \\
u \times v & =\left(v_{y} \cdot u_{z}-v_{z} \cdot u_{y}, v_{z} \cdot u_{x}-v_{x} \cdot u_{z}, v_{x} \cdot u_{y}-v_{y} \cdot u_{x}\right)
\end{aligned}
$$

Com estas informações é possível construir a matriz $M_{s a}$, conforme Equação 6 . Tendo $M_{s a}$ em mãos, o vetor correspondente a direção do eixo $\mathrm{X}$ do módulo sensor pode ser calculada pela Equação 7 .

$$
\begin{gathered}
M_{s a}=\left[\begin{array}{lll}
u_{x} & v_{y} \cdot u_{z}-v_{z} \cdot u_{y} & v_{x} \\
u_{y} & v_{z} \cdot u_{x}-v_{x} \cdot u_{z} & v_{y} \\
u_{z} & v_{x} \cdot u_{y}-v_{y} \cdot u_{x} & v_{z}
\end{array}\right] \\
M_{s a} \cdot(x, y, z)=(1,0,0)
\end{gathered}
$$

Resolvendo o sistema obtêm-se os valores $x, y \mathrm{e} z$ correspondentes às coordenadas do vetor unitário na direção do eixo X do módulo sensor na base do ambiente. Assim,

\footnotetext{
${ }^{5} \times$ é o sinal de produto vetorial
} 
para calcular o ângulo entre dois módulos sensores, determina-se a direção do eixo X de ambos na base do ambiente e calcula-se o ângulo com a Equação 1.

O procedimento descrito é ilustrado na Figura 6 para o caso bidimensional. Os retângulos representam módulos sensores, cada um com a indicação da direção de seu eixo X. Os vetores de referência, $b_{x}$ e $b_{z}$, também são representados na figura.

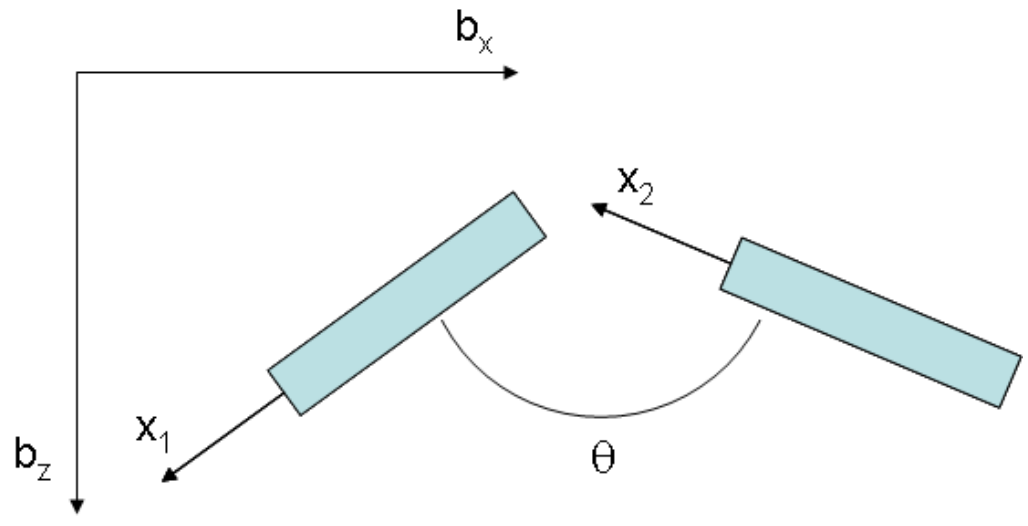

Figura 6. Exemplo 2D

Os vetores $x_{1}=\left(\alpha_{1}, \beta_{1}\right)_{b}$ e $x_{2}=\left(\alpha_{2}, \beta_{2}\right)_{b}$ são decompostos como combinações lineares da base, para algum valor de $\alpha_{1}, \alpha_{2}, \beta_{1}$ e $\beta_{2}$. O ângulo $\theta$ entre eles pode então ser calculado por $\theta=\arccos \left(\alpha_{1} \alpha_{2}+\beta_{1} \beta_{2}\right)$, para $x_{1}$ e $x_{2}$ unitários.

Para o protótipo apresentado, o sensor utilizado para aferir $b_{z}$ foi o acelerômetro. A calibração de cada um de seus eixos foi realizada através da aquisição de valores simétricos conhecidos, desta forma o offset do sensor é dado pela média aritmética de todos os valores adquiridos [Shimmer Research 2012].

Dado o uso exclusivo de acelerômetro, não foi utilizado nenhum sensor para aferir o vetor de referência horizontal, tendo sido adotado um valor constante. Devido a esta simplificação, o sistema é capaz de aferir ângulos articulares somente nos casos em que o eixo X dos módulos sensores encontram-se em um mesmo plano vertical. Esta restrição pode ser contornada a partir da adição de outros sensores, como magnetômetros e giroscópios.

\subsection{Hardware}

Dos sete módulos sensores do sistema, seis são módulos básicos Shimmer [Shimmer Research 2011], vistos na Figura 7. Estes módulos são munidos de acelerômetro de três eixos e bateria. O sétimo módulo é um dispositivo TelosB [MEMSIC Inc. 2004] que, no escopo do sistema, tem a função de sorvedouro de dados, ou seja, receptor dos dados da aplicação.

Ambos os módulos possuem características de processamento e memória semelhantes, e seguem o mesmo padrão de comunicação sem fio IEEE 802.15.4 [IEEE Standard 2006], implementado pelo microcontrolador do rádio transceptor CC2420 [Texas Instruments 2007], que além do recursos de acesso ao meio, pode fornecer serviços de segurança como confidencialidade, integridade e 
autenticidade dos dados, recurso importante para atender os requisitos de privacidade do paciente.

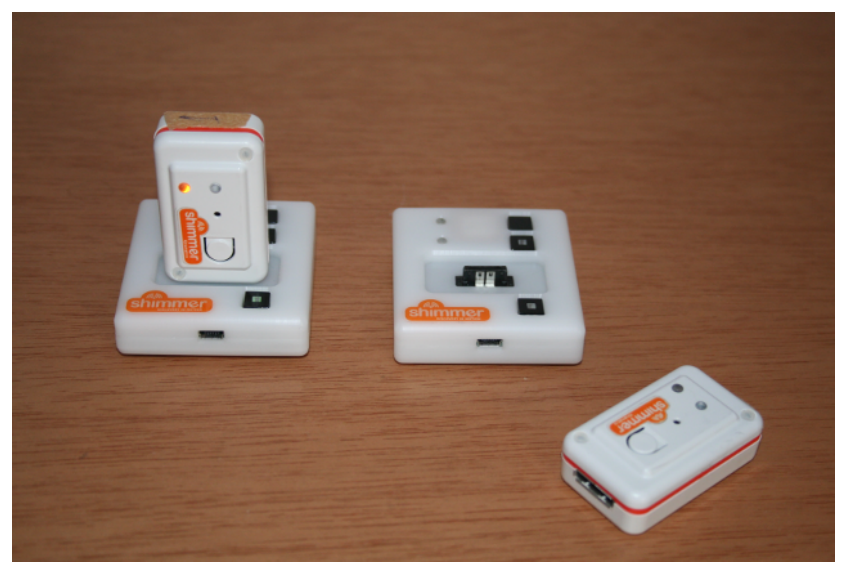

Figura 7. Módulos Sensores

\subsection{Sofware}

O software pode ser dividido em duas categorias: (i) o software embarcado desenvolvido para os módulos sensores, e (ii) o software da estação do fisioterapeuta, dividido em processamento de dados, interface gráfica e banco de dados.

\subsubsection{Software Embarcado}

Os módulos sensores utilizam o sistema operacional TinyOS [Levis et al. 2005], caracterizado por ser orientado a eventos e projetado especialmente para plataformas com recursos de memória e processamento restritos. Um programa feito para TinyOS é composto pela instanciação de componentes e a interação destes com o programa principal através de suas interfaces.

Os componentes em geral representam componentes de hardware, como módulos de comunicação ou sensores, enquanto que as interfaces definem os comandos que os componentes podem realizar. A codificação é realizada em nesC [Gay et al. 2003], uma linguagem de programação derivada do C.

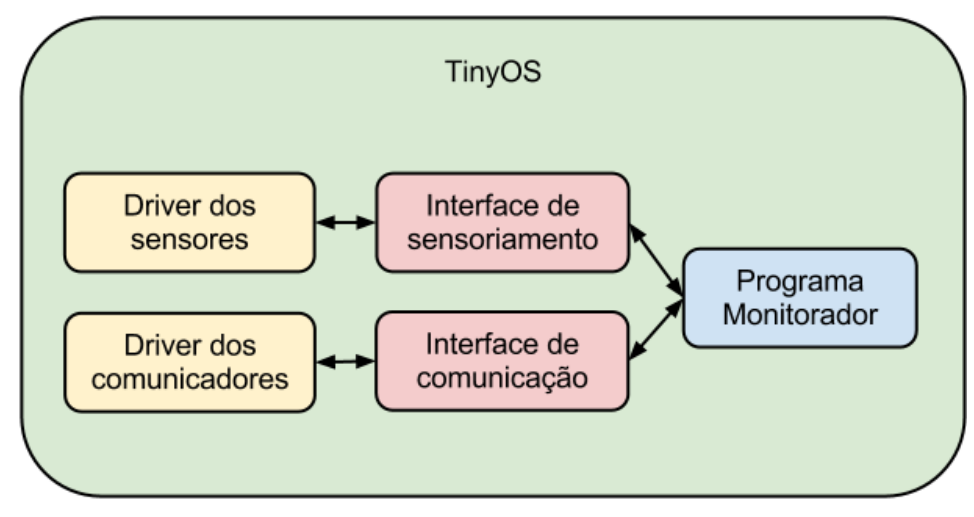

Figura 8. Arquitetura do software embarcado 
O software desenvolvido para os módulos sensores pode ser dividido em três etapas de execução distintas: inicialização dos componentes, leitura e envio de valores. A arquitetura é exibida da Figura 8.

A inicialização contempla a ativação do acelerômetro, do módulo de comunicação e de um componente de temporização responsável pelo disparo das leituras dos sensores. Após a inicialização do sistema embarcado, uma sequência periódica de leituras e envios de dados é mantida durante o uso do sistema, a cada 128 milissegundos ou de acordo com período configurado pelo usuário.

A leitura do acelerômetro é composta pela obtenção de dados referente a três eixos ortogonais, formando uma tripla de valores inteiros de 16 bits. A partir da finalização de cada leitura, os dados obtidos são encapsulados e encaminhados à interface de rádio, que por sua vez os envia para o módulo sorvedouro.

\subsubsection{Software de Processamento de Dados}

O software que calcula os resultados a partir dos dados obtidos dos módulos sensores é organizado conforme a arquitetura apresentada na Figura 9.

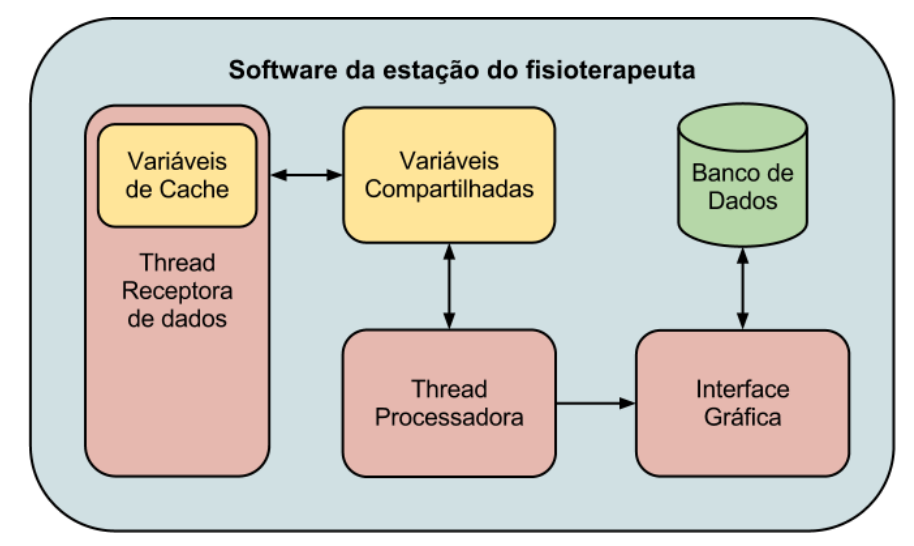

Figura 9. Arquitetura do software de processamento de dados

O software é composto basicamente por duas threads, além daquelas que controlam a interface gráfica com o usuário. Uma destas threads, intitulada "Thread Receptora", é responsável por receber os dados dos módulos sensores através do módulo sorvedouro. Além disso, é responsável por adicionar os dados recebidos ao log do sistema.

A outra thread, a "Thread Processadora", transforma os dados obtidos pela primeira, com base nos cálculos definidos da Seção 3.1, e então submete os resultados para serem exibidos na interface gráfica. Esta também exerce a função de armazenar os valor máximo, mínimo e médio ao término da sessão.

\subsubsection{Banco de dados}

O sistema utiliza o gerenciador de bancos de dados MySQL [Oracle Corporation 2011] para armazenar os dados de pacientes e tratamentos. O banco de dados é composto por 
três tabelas: Paciente, Sessão e Medição. As informações armazenadas são dados pessoais do paciente, observações feitas pela fisioterapeuta e os valores sumarizados medidos durante cada uma das sessões, conforme diagrama exibido na Figura 10.

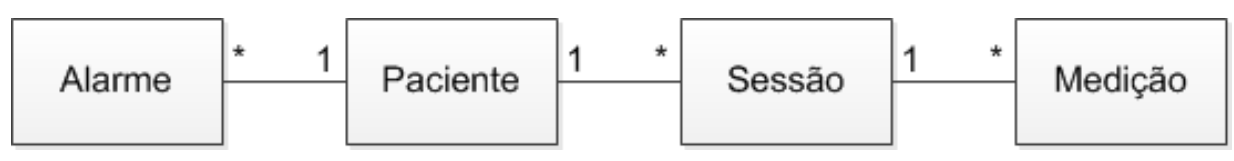

Figura 10. Diagrama entidade relacionamento

\subsection{Modelo Tridimensional}

Para melhor visualização dos dados, foi construído um modelo 3D de paciente, que é exibido em um papplet na interface gráfica. Papplet é um componente do Processing [Processing 2012], um ambiente de desenvolvimento de aplicações 3D, integrável com aplicações Java.

Tal modelo consiste de quatro cilindros, que representam os grandes ossos da perna, e um triângulo, que representa a quadril. Estes objetos são posicionados na tela de acordo com as posições calculadas dos dados dos módulos sensores, como na Figura 11.

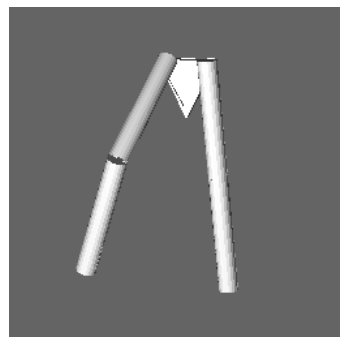

\section{Figura 11. Modelo tridimensional}

O modelo possui as seguintes funções auxiliares de visualização: zoom através do botão de rolagem do mouse; destaque de membros que formam determinados ângulos e reposicionamento da câmera com arrasto do botão direito do mouse.

\section{Resultados}

Nesta seção serão apresentados os resultados obtidos após a implementação do sistema. Estes contemplam o processo de validação das mensurações, da interface e testes com pacientes.

\subsection{Validação das Medidas do FlexMeter}

Com objetivo de averiguar a pertinência das medidas do sistema, comparou-se sua saída com ângulos estáticos determinados por um goniômetro. Quatro ângulos foram escolhidos arbitrariamente dentro do intervalo $\left[0^{\circ}, 140^{\circ}\right]$, intervalo esperado típico para uma articulação de joelho [Marques 2003].

Dois módulos sensores foram posicionados um em cada braço do goniômetro, de forma que o ângulo entre os módulos sensores fosse o mesmo que o indicado pelo instrumento. A Figura 12 ilustra este procedimento. 


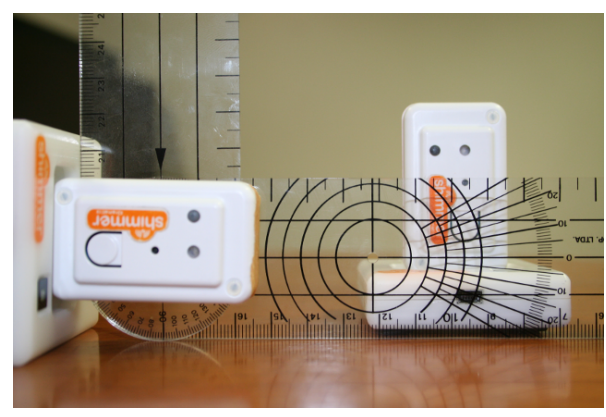

Figura 12. Posicionamento entre goniômetro e sensores

O valor retornado pelo sistema foi coletado 20 vezes para cada um dos quatro ângulos de referência escolhidos $\left(0^{\circ}, 45^{\circ}, 90^{\circ}\right.$ e $\left.120^{\circ}\right)$. Parte do erro existente nestas medidas é proveniente do erro inerente ao goniômetro e do desalinhamentos da posição relativa entre os módulos sensores e os braços do goniômetro.

Com estes dados é possível estabelecer o intervalo de confiança para cada mensuração de ângulo. Utilizando o nível de confiança de $95 \%$, assumindo que as medidas estão distribuídas de acordo com uma distribuição normal e utilizando equações clássicas obtemos a Tabela 1.

Tabela 1. Valores de mensuração do sistema - estatísticas

\begin{tabular}{|c|c|c|c|c|}
\cline { 2 - 5 } \multicolumn{2}{c|}{} & \multicolumn{4}{|c|}{ Angulos de Referência } \\
\hline Estatística & $0^{\circ}$ & $45^{\circ}$ & $90^{\circ}$ & $120^{\circ}$ \\
\hline Média & $1.695^{\circ}$ & $45.381^{\circ}$ & $90.429^{\circ}$ & $120.079^{\circ}$ \\
Comprimento do intervalo de confiança & $0.445^{\circ}$ & $0.960^{\circ}$ & $0.832^{\circ}$ & $0.695^{\circ}$ \\
Erro máximo no intervalo de confiança & $2.14^{\circ}$ & $1.34^{\circ}$ & $1.26^{\circ}$ & $0.77^{\circ}$ \\
\hline
\end{tabular}

Para considerar o erro do goniômetro na estimativa do erro do sistema, utilizase as Equações 8 e 9, para a determinação do erro médio e do desvio do erro médio, respectivamente. Desta forma, o valor pontual do erro do sistema é dado pela diferença entre o valor apontado pelo goniômetro e a média dos valores apontados pelo sistema.

$$
\begin{aligned}
& \text { Erro }_{\text {FlexMeter }}=\text { ângulo }_{\text {goniômetro }}-\overline{\text { ângulo }}{ }_{\text {FlexMeter }} \\
& \sigma_{\text {Erro }_{\text {FlexMeter }}}=\sqrt{\sigma_{\text {ângulo goniometro }^{2}}^{2}+\sigma_{\text {ângulo }_{\text {FlexMeter }}}^{2}}
\end{aligned}
$$

Foi considerado que o erro do goniômetro, $\sigma_{\text {ângulo }}{ }_{\text {goniômetro }}$, é igual a metade da menor divisão do instrumento, isto é, $1^{\circ}$. Para o erro do sistema, utilizou-se o desvio padrão da amostra, de acordo com o ângulo considerado.

Os resultados dos cálculos do erro do sistema e seu desvio encontram-se na Tabela 2. O erro médio é pequeno, porém seu desvio é grande. Contudo, de acordo com a Tabela 1, observa-se que o erro médio não é muito maior do que $2^{\circ}$, com $95 \%$ de confiança. 
Tabela 2. Valores de erro do sistema

\begin{tabular}{|c|c|c|}
\hline Ângulo & Erro & Desvio do Erro \\
\hline $0^{\circ}$ & $1.7^{\circ}$ & $1.4^{\circ}$ \\
$45^{\circ}$ & $0.4^{\circ}$ & $2.4^{\circ}$ \\
$90^{\circ}$ & $0.4^{\circ}$ & $2.1^{\circ}$ \\
$120^{\circ}$ & $0.1^{\circ}$ & $1.9^{\circ}$ \\
\hline
\end{tabular}

\subsection{Usabilidade}

Com a finalidade de avaliar a usabilidade do sistema, a fisioterapeuta co-autora utilizou o protótipo em ambiente clínico. Após a leitura da versão preliminar do manual de usuário e as funções fornecidas pelo sistema foram testadas. Notou-se a falta de algumas explicações básicas, porém necessárias para um usuário que não havia entrado em contato com o sistema anteriormente.

Quanto ao protocolo de uso, trecho do manual que indica ao seu leitor como obter a medida dos ângulos desejados, o manual trouxe os procedimentos necessários. Porém foi preciso explicitar que alguns ângulos, como os de flexão e extensão do joelho, são medidos com o mesmo par de módulos sensores e a diferenciação entre eles fica a cargo do usuário.

Não foram observados bugs ou comportamentos inesperados durante a operação. Após o entendimento dos pontos deficientes do manual, o usuário foi capaz de utilizar o sistema, seguir as etapas de preparação e realizar a mensuração do ângulo de flexão do joelho direito e observar os dados obtidos.

\subsection{Validação em pacientes}

Nas Seções 4.1 e 4.2 demostrou-se que o sistema é capaz de aferir ângulos com acurácia da mesma ordem de grandeza de um goniômetro e sua interface apresenta usabilidade para os usuários, respectivamente.

Contudo, para validar o sistema do ponto de vista da fisioterapia, é necessário a análise da mensuração da ADM em pacientes, e a comparação do resultado obtido com um método de mensuração já validado na fisioterapia. Aqueles que se sujeitaram aos experimentos foram os próprios autores devido ao escopo limitado da validação, ainda sem autorização de validação clínica em maior escala pelo comitê de ética.

Os experimentos de mensuração foram realizados na articulação do joelho de acordo com procedimento de medição padrão de fisioterapia [Marques 2003, Amado and Maria 2006], com o paciente deitado em decúbito dorsal. Para realizar a flexão, o quadril deve ficar em $90^{\circ}$, evitando a sua rotação. A extensão do joelho é o retorno da posição de flexão no plano sagital.

Os módulos sensores foram posicionados conforme procedimento de goniometria [Amado and Maria 2006]. O sensor inferior foi posicionado paralelo à face lateral da fíbula dirigido para o maléolo lateral. O outro módulo sensor foi colocado paralelo à superfície lateral do fêmur, dirigido para o trocânter maior. A distância dos módulos sensores ao joelho (côndilos da tíbia e fêmur) foi grande o suficiente para não interferir da medida do goniômetro. Um exemplo de posicionamento é exibido na Figura 13. As 
mensurações foram registradas com fotografias à distância de $1,5 \mathrm{~m}$ e à altura de $1 \mathrm{~m}$ do chão.

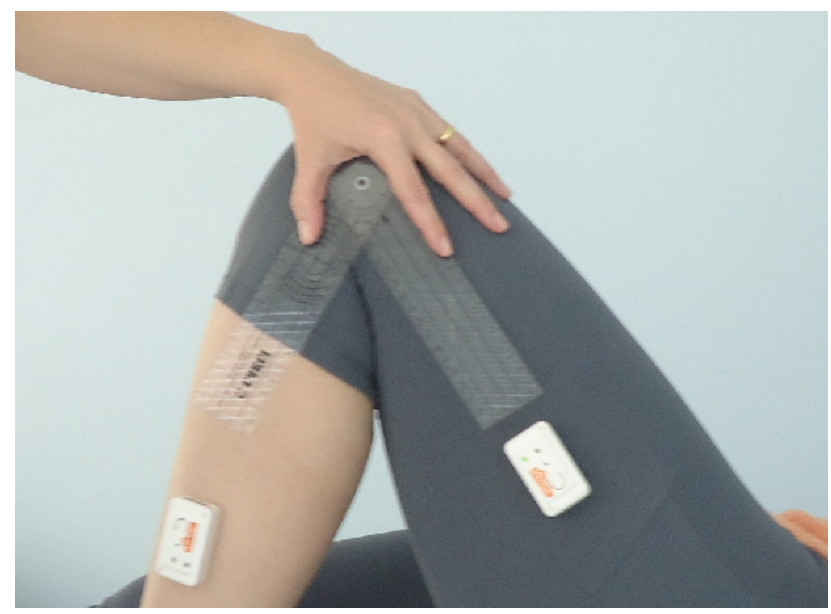

Figura 13. Posicionamento de módulos sensores e goniômetro

Em testes preliminares, para acoplar os módulos ao pacientes, estes eram colocados em um envólucro de tecido e fixados com velcro. Porém verificou-se que este método causou grandes erros nas medições. Por esta razão a fixação dos módulos sensores nos pacientes foi realizada através de fita adesiva dupla-face.

Para cada paciente foram realizadas dez medições de flexão e extensão de cada joelho com o goniômetro. Concomitantemente, foram realizadas leituras dos valores instantâneos do sistema FlexMeter.

A Tabela 3 sumariza os resultados obtidos seguindo o procedimento detalhado ao longo desta seção. É possível notar que, em geral, a medição através do goniômetro apresenta maior desvio padrão que a do sistema, indicando maior consistência nas medições.

Tabela 3. Ângulos articulares

\begin{tabular}{|ccc|cc|cc|}
\hline \multirow{2}{*}{ Paciente } & Movimento & Lado & $\begin{array}{c}\text { Média } \\
\text { Goniômetro }\end{array}$ & $\begin{array}{c}\text { Desvio } \\
\text { Padrão }\end{array}$ & $\begin{array}{c}\text { Média } \\
\text { FlexMeter }\end{array}$ & $\begin{array}{c}\text { Desvio } \\
\text { Padrão }\end{array}$ \\
\hline \hline 1 & flexão & D & 132,5 & 2,2 & 137,9 & 1,8 \\
1 & flexão & E & 128,6 & 3,7 & 119,0 & 2,4 \\
1 & extensão & D & 12,9 & 2,6 & 11,0 & 1,2 \\
1 & extensão & E & 10,1 & 0,7 & 8,1 & 0,4 \\
\hline \hline 2 & flexão & D & 136,2 & 2,3 & 139,9 & 0,9 \\
2 & flexão & E & 138,9 & 0,7 & 137,5 & 1,8 \\
2 & extensão & D & 8,0 & 2,4 & 4,6 & 1,9 \\
2 & extensão & E & 4,3 & 3,3 & 3,0 & 0,7 \\
\hline \hline 3 & flexão & D & 132,2 & 1,3 & 128,9 & 3,1 \\
3 & flexão & E & 129,8 & 1,7 & 134,7 & 5,4 \\
3 & extensão & D & 3,6 & 2,6 & 6,8 & 1,1 \\
3 & extensão & E & 2,8 & 1,2 & 2,4 & 0,7 \\
\hline
\end{tabular}

Análises estatísticas foram realizadas e os resultados são exibidos na Tabela 4. A 
diferença média entre os pares de valores obtidos com cada instrumento resulta do erro médio da medição da articulação. Em comparação com os testes realizados com goniômetro fixo (Seção 4.1), o erro constatado foi maior, o que é esperado devido à incoerências de posicionamento, e o desvio padrão manteve-se na mesma ordem de grandeza. Entretanto, 10 dos 12 casos mostram erro inferior a $5^{\circ}$, que é uma diferença admissível em termos de fisioterapia [Hayes 1992, Bertolini et al. 2008].

Tabela 4. Ângulos articulares

\begin{tabular}{|ccccccc|}
\hline Paciente & Movimento & Lado & $\begin{array}{c}\text { Erro Médio } \\
\text { (valor absoluto) }\end{array}$ & $\begin{array}{c}\text { Desvio padrão } \\
\text { do erro }\end{array}$ & P & $\begin{array}{c}\text { Rejeita } \\
H_{0}\end{array}$ \\
\hline \hline 1 & flexão & $\mathrm{D}$ & 5,4 & 2,8 & 0,006 & Sim \\
1 & flexão & $\mathrm{E}$ & 9,6 & 2,9 & 0,006 & Sim \\
1 & extensão & $\mathrm{D}$ & 1,9 & 3,1 & 0,074 & Não \\
1 & extensão & $\mathrm{E}$ & 2,0 & 0,7 & 0,006 & Sim \\
\hline \hline 2 & flexão & $\mathrm{D}$ & 3,7 & 2,7 & 0,009 & Sim \\
2 & flexão & $\mathrm{E}$ & 1,4 & 1,8 & 0,056 & Não \\
2 & extensão & $\mathrm{D}$ & 3,4 & 2,1 & 0,008 & Sim \\
2 & extensão & $\mathrm{E}$ & 1,3 & 3,1 & 0,236 & Não \\
\hline \hline 3 & flexão & $\mathrm{D}$ & 3,3 & 3,5 & 0,028 & Sim \\
3 & flexão & $\mathrm{E}$ & 4,9 & 4,5 & 0,021 & Sim \\
3 & extensão & $\mathrm{D}$ & 3,2 & 2,0 & 0,008 & Sim \\
3 & extensão & $\mathrm{E}$ & 0,4 & 0,9 & 0,212 & Não \\
\hline
\end{tabular}

O valor $\mathrm{P}$ exibido na tabela foi calculado utilizando o Teste de Wilcoxon [Wilcoxon 1945], um teste estatístico não paramétrico para dados pareados. O teste é feito sobre a hipótese nula $\left(H_{0}\right)$ de que a diferença entre as amostra é igual a zero. A hipótese $H_{0}$ é rejeitada se o valor calculado de $\mathrm{P}$ foi inferior ao nível de significância $\alpha$, que para o nível de confiança adotado de $95 \%$ é igual a 0,05 . Os cálculos foram realizados através da ferramenta estatística R [The R Project 2012].

Tabela 5. Ângulos articulares

\begin{tabular}{|ccc|}
\hline Agrupamento & $\mathrm{P}$ & Rejeita $H_{0}$ \\
\hline \hline Paciente 1 flexão & 0,167 & Não \\
Paciente 2 flexão & 0,190 & Não \\
Paciente 3 flexão & 0,632 & Não \\
Flexão - todos os pacientes & 0,911 & Não \\
Paciente 1 extensão & 0,001 & Sim \\
Paciente 2 extensão & 0,004 & Sim \\
Paciente 3 extensão & 0,038 & Sim \\
Extensão - todos os pacientes & 0,010 & Sim \\
Todas as medições & 0,273 & Não \\
\hline
\end{tabular}

Neste nível de confiança a hipótese nula é rejeitada dois terços dos casos, que são os que possuem os maiores erros médios. O resultado desfavorável na maioria dos casos deve-se ao número reduzido de amostras do ponto de vista estatístico e a sensibilidade do 
teste empregado a amostras pequenas [Feltovich 2003]. No intuito de evitar esta sensibilidade, a massa de dados foi agrupada de acordo com o tipo de medição conforme indicado na Tabela 5. Nota-se que somente as medições relacionadas a extensão apresentam resultado desfavorável. Contudo, quando todo o conjunto de medições é analisado, verifica-se que o resultado do teste é positivo.

Portanto, os resultados obtidos através do processo de validação de mensuração dos ângulos e os avaliação de usabilidade realizada pela fisioterapeuta demostram o potencial do sistema como aplicação prática.

\section{Considerações Finais}

No contexto das cidades inteligentes, desenvolver ferramentas de apoio a fisioterapia permite expandir o suporte a área de saúde. Este trabalho é resultado da intersecção entre a engenharia e fisioterapia, proporcionando maior automação e poder de processamento de dados na tarefa de monitoramento de amplitude de movimento através do sistema proposto.

O protótipo descrito e validado neste artigo realiza a mensuração de ângulos articulares em tempo real, através de dispositivos de sensoriamento conectados por redes redes sem fio, garantindo a mobilidade do paciente e do fisioterapeuta. Os dados que o sistema armazena durante as sessões permitem ao fisioterapeuta acompanhar a evolução do paciente e gerar relatórios clínicos completos, permitindo quantificar a efetividade do tratamento.

O processo de validação constatou que o erro cometido pelo sistema ao realizar medições de ângulos articulares é da mesma ordem de grandeza do goniômetro. O teste de usabilidade realizado contemplou a implantação preliminar do sistema em ambiente clínico. A terceira etapa da validação incluiu o uso do sistema para medir ângulos articulares do joelho, e sua comparação com medidas obtidas com o goniômetro universal. Embora a comparação individual das amostras com o teste de Wilcoxon (com nível de confiança de $95 \%$ ) tenha rejeitado a hipótese nula na maioria dos casos, o teste aponta resultado favorável quando as medições são agregadas. Os resultados das três etapas de validação do sistema demonstraram como seu uso pode facilitar o trabalho do fisioterapeuta e o potencial da aplicação do sistema.

Como discutido na Seção 3.1, a adição de outros sensores, como magnetômetros e giroscópios, permitirão ao sistema aferir ângulos articulares em qualquer posição do paciente. Neste caso, o sistema poderia ser utilizado como ferramenta de suporte e acompanhamento em educação física, após a adição de filtros para lidar com as grandes acelerações envolvidas. Além disso, pretende-se estender os testes realizados, reproduzindo o experimento em diversos pacientes e com maior número de fisioterapeutas.

\section{Agradecimentos}

Bruno Trevizan de Oliveira possui Bolsa de Mestrado financiada pela Fundação de Amparo à Pesquisa do Estado de São Paulo, processo FAPESP 2010/02909-8.

\section{Referências}

[Alves et al. 2012] Alves, R. C. A., Santos, F., de Oliveira, B. T., and Margi, C. B. (2012).

Sistema de monitoramento de amplitude de movimento baseado em redes de sensores 
sem fio aplicado à fisioterapia. In Anais do VIII Simpósio Brasileiro de Sistemas de Informação, pages 504-515.

[Amado and Maria 2006] Amado, J. and Maria, S. (2006). Métodos de avaliação clínica e funcional em fisioterapia. Guanabara Koogan.

[Batistela 2008] Batistela, R. A. (2008). Contribuição da amplitude de movimento articular do joelho na marcha de crianças com paralisia cerebral espástica. Trabalho de conclusão de curso. Universidade Estadual Paulista, Instituto de Biociências de Rio Claro.

[Bennell et al. 1998] Bennell, K., Talbot, R., Wajswelner, H., Techovanich, W., Kelly, D., and Hall, A. (1998). Intra-rater and inter-rater reliability of a weight-bearing lunge measure of ankle dorsiflexion. Aust J Physiother, 44(3):175-180.

[Bertolini et al. 2008] Bertolini, G. R. F., Filippin, T. R., Onishi, C. M., Ariza, D., Nakayama, G. K., and Loth, E. A. (2008). Avaliação dos métodos de alongamento estático e alongamento estático combinado ao ultra-som na extensibilidade do gastrocnêmico. Fisioter. mov., 1(21):115-122.

[Biometrics Ltd 2012] Biometrics Ltd (2012). Goniometer \& Torsiometer. http : / www . biometricsltd.com/gonio.htm.

[BioSmart 2012] BioSmart (2012). Biofeed. http://www.biosmart.com.br/ biofeed.html.

[Canto et al. 2005] Canto, R., Anjos Filho, G. S., Magalhães, L., Moreira, M. Q., Canto, F. R. D. T., Baraúna, M. A., Sanchez, H. M., and Silva, R. A. V. (2005). Anteversão do colo do fêmur: avaliação clínica versus radiológica. Acta ortop. bras., 13(4):171-174. ISSN 1413-7852.

[CardioMed 2012] CardioMed (2012). Flexímetro. http: / /www. cardiomed.com. br/fleximetro.html.

[Carnaz 2008] Carnaz, L. (2008). Eletrogoniometria flexível: Avaliação da simetria articular e dos efeitos dos sensores, das coletas e dos joelhos em registros da marcha. Master's thesis, Universidade Federal de São Carlos.

[Clarkson and Gilewich 1991] Clarkson, H. and Gilewich, G. (1991). Avaliaçao musculoesqueletica: amplitude de movimento articular e força muscular manual. Manole.

[COFFITO 2012] COFFITO (2012). Definição de fisioterapia. Conselho Federal de Fisioterapia e Terapia Ocupacional.

[Culler et al. 2004] Culler, D., Estrin, D., and Srivastava, M. (2004). Overview of sensor networks. Computer Magazine, 37(8):41-49.

[de Winter et al. 2004] de Winter, A. F., Heemskerk, M. A. M. B., Terwee, C. B., Jans, M. P., Devillé;, W., van Schaardenburg, D.-J., Scholten, R. J. P. M., and Bouter, L. M. (2004). Inter-observer reproducibility of measurements of range of motion in patients with shoulder pain using a digital inclinometer. BMC Musculoskelet Disord, 5:18.

[Della Mea 2001] Della Mea, V. (2001). What is e-health (2): The death of telemedicine? $J$ Med Internet Res, 3(2):e22.

[EMG System do Brail 2012] EMG System do Brail (2012). Goniômetro. http: / / www . emgsystem.com.br/produtos/goniometro. 
[Feltovich 2003] Feltovich, N. (2003). Nonparametric tests of differences in medians: Comparison of the wilcoxonmann-whitney and robust rank-order tests. Experimental Economics, pages 6-273.

[Fysio Brasil 2012] Fysio Brasil (2012). Fysio brasil. http://www. fysiobrasil. com.

[Gajdosik and Bohannon 1987] Gajdosik, R. L. and Bohannon, R. W. (1987). Clinical measurement of range of motion. review of goniometry emphasizing reliability and validity. Phys Ther, 67(12):1867-1872.

[Gay et al. 2003] Gay, D., Welsh, M., Levis, P., Brewer, E., Behren, R. V., and Culler, D. (2003). The nesc language: A holistic approach to networked embedded systems. In In Proceedings of Programming Language Design and Implementation (PLDI, pages $1-11$.

[Hayes 1992] Hayes, K. W. (1992). The effect of awareness of measurment error on physical therapists' confidence in their decisions. Physical Therapy, 72(7):515-525.

[IEEE Standard 2006] IEEE Standard (2006). IEEE 802.15.4: Wireless medium access control (MAC) and physical layer (PHY) specifications for low-rate wireless personal area networks (WPANs).

[Levis et al. 2005] Levis, P., Madden, S., Polastre, J., Szewczyk, R., Whitehouse, K., Woo, A., Gay, D., Hill, J., Welsh, M., Brewer, E., and Culler, D. (2005). TinyOS: an operating system for sensor networks ambient intelligence. In Weber, W., Rabaey, J. M., and Aarts, E., editors, Ambient Intelligence, chapter 7, pages 115-148. Springer Berlin Heidelberg, Berlin/Heidelberg.

[Lorincz et al. 2009] Lorincz, K., Chen, B.-r., Challen, G. W., Chowdhury, A. R., Patel, S., Bonato, P., and Welsh, M. (2009). Mercury: a wearable sensor network platform for high-fidelity motion analysis. In Proceedings of the 7th ACM Conference on Embedded Networked Sensor Systems, SenSys '09, pages 183-196, New York, NY, USA. ACM.

[Malan et al. 2004] Malan, D., Fulford-jones, T., Welsh, M., and Moulton, S. (2004). Codeblue: An ad hoc sensor network infrastructure for emergency medical care. In International Workshop on Wearable and Implantable Body Sensor Networks.

[Margi 2006] Margi, C. B. (2006). Energy Consumption Trade-offs In Power Constrained Networks. PhD thesis, University of California Santa Cruz.

[Marques 2003] Marques, A. (2003). Manual de goniometria. Manole, segunda edition.

[MEMSIC Inc. 2004] MEMSIC Inc. (2004). telosB product details - 6020-0094-04 Rev B. http://www.memsic.com/support/documentation/wirelesssensor-networks/category/7-datasheets.html?download=152\% 3Atelosb.

[O'Donovan and Ayer 2011] O'Donovan, K. and Ayer, S. (2011). Real-time joint angle measurement using the shimmer wireless sensor platform. In Proceedings of the First ACM Workshop on Mobile Systems, Applications, and Services for Healthcare, mHealthSys '11, pages 7:1-7:2, New York, NY, USA. ACM.

[Oracle Corporation 2011] Oracle Corporation (2011). Mysql website. http://www . mysql. com/. 
[Polizel et al. 2011] Polizel, A. S., Wada, E. D., and Alves, R. C. A. (2011). Redes de sensores sem fio aplicadas à fisioterapia. Tese de conclusão de curso, Escola Politécnica, Universidade de São Paulo, São Paulo.

[Portney and Watkins 2000] Portney, L. and Watkins, M. (2000). Foundations of clinical research: applications to practice. Prentice Hall Health.

[Processing 2012] Processing (2012). Processing Website. http://processing . org/.

[Rothstein 1985] Rothstein, J. (1985). Measurement in physical therapy. Clinics in physical therapy. Churchill Livingstone.

[Shimmer Research 2011] Shimmer Research (2011). Shimmer - wireless sensor platform. http://www.shimmer-research.com/wp-content/uploads/ 2011/05/Shimmer-Platform.pdf.

[Shimmer Research 2012] Shimmer Research (2012). 9DOF calibration user manual - Rev 1.0b. http://www.shimmer-research.com/wp-content/ uploads/2012/02/Shimmer-9DoF-Calibration-User-Manual.pdf.

[Texas Instruments 2007] Texas Instruments (2007). Chipcon cc2420 datasheet. http: // inst. eecs.berkeley. edu/ cs150/Documents/CC2420.pdf.

[The R Project 2012] The R Project (2012). The R Project for Statistical Computing Website. http://www.r-project.org/.

[Wilcoxon 1945] Wilcoxon, F. (1945). Individual Comparisons by Ranking Methods. Biometrics Bulletin, 1(6):80-83.

[Williams et al. 1993] Williams, R., Binkley, J., Bloch, R., Goldsmith, C. H., and Minuk, T. (1993). Reliability of the modified-modified schöber and double inclinometer methods for measuring lumbar flexion and extension. Physical Therapy, 73(1):26-37. 